\title{
The differences between the Connor-Davidson Resilience Scale and the Brief Resilience Scale when assessing resilience: Confirmatory factor analysis and predictive effects
}

\author{
Yun-Ci Ye ${ }^{1}$, Chia-Huei $\mathrm{Wu}^{2,3}$, Chang-Ta Yang ${ }^{4,5}$ \\ 1 Department of Somatics and Sport Leisure Industry, College of Humanities, National Taitung University, \\ Taitung, Taiwan \\ 2 Management Department, Leeds University Business School, University of Leeds, UK \\ 3 Department of Medical Research, China Medical University Hospital, China Medical University, Taichung, \\ Taiwan \\ 4 Graduate Institute of Mind, Brain and Consciousness, Taipei Medical University, Taipei, Taiwan \\ 5 Department of Psychology, National Cheng Kung University, Tainan, Taiwan \\ * Correspondence: CT Yang: yangct@mail.ncku.edu.tw; YC Ye: hayoki@gmail.com
}

\begin{abstract}
The Connor-Davidson Resilience Scale (CD-RISC) and the Brief Resilience Scale (BRS) are two widely used scales to measure resilience. Although both scales seek to assess an individual's ability to recover from and adapt to disruptions or stressful events, they may capture different aspects of resilience. While the CD-RISC focuses on resources that can help individuals recover from and adapt to disruptions or stressful events, the BRS directly measures one's ability to bounce back or be resilient. The aim of this study is to empirically examine the differences between the CD-RISC and the BRS. Two samples $(n=202,246)$ consisting of undergraduate students from Taiwan were used. The results of confirmatory factor analysis show that the CD-RISC and the BRS are highly correlated but still distinct. The results of regression analyses show that the CD-RISC and the BRS have unique predictive effects on depression and life satisfaction. The research findings suggest that the CD-RISC and the BRS capture different aspects of resilience. For future research on resilience, researchers should pay attention to the differences between these scales and choose the one that most closely fits their research purpose.
\end{abstract}

Keywords: Resilience, Connor-Davidson Resilience Scale, Brief Resilience Scale, Depression, Life satisfaction, Confirmatory factor analysis

\section{Introduction}

Individual resilience has been recognized as an important topic in the behavioral and medical sciences $[1,2]$. During the past decade, resilience has been conceptualized from different angles, and different tools have been developed to assess resilience in different ways [3] Among the available tools to measure resilience, the Connor-Davidson Resilience Scale (CD-RISC) [4] and the Brief Resilience Scale (BRS) [5] are two widely used scales. Although both tools seek to assess an individual's ability to recover from and adapt to disruptions or stressful events, they are different in several ways.

Specifically, resilience is considered a multidimensional concept in the CD-RISC [4]. This scale assesses resilience by examining five dimensions: (1) personal competence, (2) trust in one's instincts, tolerance of negative affect, and strengthening effects of stress, (3) positive acceptance of change, (4) sense of control, and (5) spiritual influence. The CD-RISC not only captures one's own ability but also covers one's attitude and access to external support (such as social support under the control dimension and spiritual forces 
as the final dimension). In contrast, resilience is conceptualized as a unidimensional concept in the BRS [5]. As indicated by Smith et al. [5], they seek to "clarify the study of resilience by presenting a scale for assessing the original and most basic meaning of the word resilience" and develop the BRS to "assess the ability to bounce back or recover from stress". As such, while the CD-RISC focuses on internal and external factors that can help individuals recover from and adapt to disruptions or stressful events, the BRS directly measures one's ability to bounce back or be resilient. These two measurements thus differ not only in their length and measurement structure but also in the definition of resilience. We argue that both conceptualizations of resilience are equally important to prevent one's illness and promote psychological wellbeing. This is the case because to recover from adversity, one will not only need to have their own psychological resources or external resources to cope with the situations [6,7] but also need to have a strong belief that they are capable of bouncing back from difficult situations [5].

The aim of this study is to examine the differences between the CD-RISC and the BRS in two steps. First, we perform confirmatory factor analysis to examine the relationships between the two measurements. To date, studies have only reported their simple correlations [5,8] without examining their factor structures simultaneously. Without correcting measurement errors and controlling for potential wording effects due to the inclusion of negative-worded items [9] in the BRS, the correlation between the CD-RISC and the BRS cannot be properly assessed. We thus aim to examine the relationships between the CD-RISC and the BRS based on the latent factor extracted from each measurement. Second, to examine our hypothesis that the CD-RISC and the BRS capture different aspects of resilience that are important for wellbeing, we use the CD-RISC and the BRS to predict depression and life satisfaction, a negative and a positive indicator of well-being, respectively. We expect that both the CD-RISC and the BRS will have significant predictive effects on depression and life satisfaction. We used two samples in this study for cross-validation.

\section{Materials and Methods}

\subsection{Participants and procedure}

We used two samples consisting of undergraduate students from two universities in Taiwan and participants from the community. Some of the participants were the students who took the introductory psychology course at National Cheng Kung University and would receive course credit for their participation, and the other participants were recruited online and were reimbursed with NTD 150 for their participation. Sample 1 included 202 participants. They were invited to participate in this study in 2019. The mean age of the participants was 20.19 years $(S D=1.31)$, and $63.4 \%$ were female. Sample 2 included 246 participants. They were invited to participate in this study in 2020 . The mean age was 19.67 years $(\mathrm{SD}=1.25)$, and most participants were male $(61.2 \%)$. As faceto-face commerce was still allowed in Taiwan, data collection for this study was not affected by COVID-19.

\subsection{Measurements}

\subsubsection{Brief Resilience Scale (BRS)}

The BRS consists of 6 items [5]. Each item was rated on a 5-point scale ranging from $1=$ "strongly disagree" to $5=$ "strongly agree". We used the traditional Chinese version validated by [10]. An average score is used to indicate the level of resilience. The Cronbach's alpha coefficient was 0.76 and 0.79 in Samples 1 and 2, respectively.

\subsubsection{The Connor-Davidson Resilience Scale (CD-RISC)}


The CD-RISC evaluates the psychological trait that exhibits resilience ${ }^{5}$. The questionnaire contains 25 items encompassing five dimensions: personal competence, trust, positive acceptance, control, and spiritual influence. Items were rated on a 5-point scale ranging from not true at all (0) to true nearly all of the time (4). A total score was used to indicate greater resilience. We used a traditional Chinese version validated by Wang et al. [11]. The Cronbach's alpha coefficient was 0.93 and 0.91 in Samples 1 and 2, respectively.

\subsubsection{Beck Depression Inventory second edition (BDI-II)}

The BDI-II was used to assess depression [12]. The scale consists of 21 items calculated on a 4-point scale from 0 to 3 . After summing all items, the total score ranged from 0 to 63 , with higher scores indicating a higher level of depression. We used a traditional Chinese version. Its reliability and validity have been reported by [13]. The Cronbach's alpha coefficient was 0.92 and 0.88 in Samples 1 and 2, respectively.

\subsubsection{Satisfaction with life scale (SWLS)}

The SWLS measures an individual's cognitive perception of subjective wellbeing [14]. The scale consists of 5 items rated on a 7-point scale from strongly disagree (1) to strongly agree (7). A total score is used to indicate a high level of SWLS. The traditional Chinese version of the SWLS has been validated by Wu and Yao [15]. The Cronbach's alpha coefficient was 0.91 and 0.91 in Samples 1 and 2, respectively.

\section{Results}

Table 1 presents the descriptive statistics of the research variables. We first performed a confirmatory factory analysis (CFA) to validate the association between the latent factor of the BRS and the latent factor of the CD-RISC for both samples. In the CFA model, the latent factor of the CD-RISC was indicated by five dimensions, and the latent factor of BRS was indicated by six items. We used dimensions, but not items, as the indicators for the CD-RISC to simplify its measurement structure because our aim is to examine the correlations between the latent factors of the BRS and the CD-RISC. We also specified a method factor indicated by three negatively worded items in the BRS. This method factor can help partial out variances of negatively wording effect when we extract latent factors of the BRS and the CD-RISC.

We estimated the model using Mplus 7.3 with the maximum likelihood estimator [16]. Figures 1 and 2 present standardized factor loadings of the hypothesized CFA model in each sample. In Sample 1, the model was acceptable $\left(\chi^{2}=94.09, \mathrm{df}=40 ; \mathrm{CFI}=\right.$ $.95, \mathrm{TLI}=.93, \mathrm{RMSEA}=.082$, SRMR $=.054)$. All estimates in the model were significant at $\mathrm{p}<.001$. The latent factor of the BRS was positively related to the latent factor of the CDRISC $(\gamma=.79, \mathrm{p}<.001)$. We also examined a model which has a latent factor influencing all items of the BRS and the five dimensions of the CD-RISC and a method factor influencing the three negatively worded items in the BRS. This model $\left(\chi^{2}=145.84, \mathrm{df}=41\right.$; $\mathrm{CFI}=.90, \mathrm{TLI}=.87$, RMSEA $=.11$, SRMR $=.060$ ) was worse than the model with two latent factors and one method factor $\left(\Delta \chi^{2}=51.75, \mathrm{df}=1, \mathrm{p}<.001\right)$.

In Sample 2, the model with two latent factors and one method was also acceptable $\left(\chi^{2}=88.04, \mathrm{df}=40 ; \mathrm{CFI}=.96, \mathrm{TLI}=.95, \mathrm{RMSEA}=.07, \mathrm{SRMR}=.042\right)$. All factor loadings were significant $(\mathrm{p}<.001)$. The latent factor of the BRS was also positively related to the latent factor of the CD-RISC $(\gamma=.83, \mathrm{p}<.001)$. We also examined a model which has only a latent factor for all indicators and a method factor influencing the three negatively worded items. This model $\left(\chi^{2}=140.04, \mathrm{df}=41 ; \mathrm{CFI}=.92, \mathrm{TLI}=.89, \mathrm{RMSEA}=.10\right.$, SRMR $=$ .055) was worse than the model with two latent factors and one method factor $\left(\Delta \chi^{2}=52\right.$, $\mathrm{df}=1, \mathrm{p}<.001$ ). 
To examine whether the BRS and the CD-RISC have unique effect in predicting the BDI-II and the SWLS, we performed regression analysis and presented the results in Table 2. In Sample 1, we found that both the BRS and CD-RISC negatively predicted scores on the BDI-II $(\beta=-.30, \mathrm{p}<.001 ; \beta=-.30, \mathrm{p}<.001)$ and positively predicted scores on the SWLS $(\beta=.18, \mathrm{p}<.001 ; \beta=.34, \mathrm{p}<.05)$. We then performed hierarchical regression analysis to examine incremental predictive effect of the two measures on the BDI-II and the CD-RISC. As reported in Table 3, we found that the BRS explained additional variances of the BDI-II $\left(\Delta \mathrm{R}^{2}=.05, \mathrm{~F}(1,199)=14.55, \mathrm{p}<.001\right)$ and the SWLS $\left(\Delta \mathrm{R}^{2}=.02, \mathrm{~F}(1,199)=\right.$ $4.59, \mathrm{p}<.05)$ beyond the CD-RISC. We also found that the CD-RISC explained additional variances of the BDI-II $\left(\Delta \mathrm{R}^{2}=.05, \mathrm{~F}(1,199)=15.13, \mathrm{p}<.001\right)$ and the SWLS $\left(\Delta \mathrm{R}^{2}=.07, \mathrm{~F}(1\right.$, $199)=17.22, \mathrm{p}<.001)$ beyond the CD-RISC.

In Sample 2, both the BRS and CD-RISC also negatively predicted scores on the BDI-II $(\beta=-.29, \mathrm{p}<.001 ; \beta=-.37, \mathrm{p}<.001)$ and positively predicted scores on the SWLS $(\beta$ $=.13, \mathrm{p}<.001 ; \beta=.56, \mathrm{p}<.05$ ). We performed hierarchical regression analysis (see Table $3)$ and found that the BRS explained additional variances of the BDI-II $\left(\Delta R^{2}=.05, F(1\right.$, $243)=19.26, \mathrm{p}<.001)$ and the SWLS $\left(\Delta \mathrm{R}^{2}=.01, \mathrm{~F}(1,243)=4.01, \mathrm{p}<.05\right)$ beyond the CDRISC. We also found that the CD-RISC explained additional variances of the BDI-II $\left(\Delta \mathrm{R}^{2}\right.$ $=.09, \mathrm{~F}(1,243)=32.49, \mathrm{p}<.001)$ and the SWLS $\left(\Delta \mathrm{R}^{2}=.18, \mathrm{~F}(1,243)=74.38, \mathrm{p}<.001\right)$ beyond the BRS.

\subsection{Figures, Tables and Schemes}

Table 1. Descriptive statistics and Pearson correlations between all variables.

\begin{tabular}{|c|c|c|c|c|c|c|}
\hline Sample 1 & & & & & & \\
\hline Variable & $M$ & $S D$ & 1 & 2 & 3 & 4 \\
\hline 1.CD-RISC & 62.08 & 13.72 & --- & & & \\
\hline 2.BRS & 3.11 & 0.63 & $.65^{* *}$ & --- & & \\
\hline 3.BDI-II & 11.51 & 9.51 & $-.49^{* *}$ & $-.49^{* *}$ & & \\
\hline 4.SWLS & 20.79 & 6.57 & $.45^{* *}$ & $.39^{* *}$ & $-.48^{* *}$ & --- \\
\hline \multicolumn{7}{|l|}{ Sample 2} \\
\hline Variable & $M$ & $S D$ & 1 & 2 & 3 & 4 \\
\hline 1.CD-RISC & 62.59 & 12.11 & -- & & & \\
\hline 2.BRS & 3.24 & 0.57 & $.62^{* *}$ & --- & & \\
\hline 3.BDI-II & 9.38 & 7.26 & $-.55^{* *}$ & $-.52^{* *}$ & --- & \\
\hline 4.SWLS & 21.85 & 6.34 & $.62^{* *}$ & $.46^{* *}$ & $-.63^{* *}$ & --- \\
\hline
\end{tabular}

${ }^{1}$ Notes: $N=202$ for Study 1; N= 246 for Study 2 .

${ }^{* *} p<.01$. 
Table 2. Standardized coefficients of multiple regression in Sample 1 and 2.

\begin{tabular}{lllll}
\hline & \multicolumn{2}{c}{ Sample 1 $(\mathrm{n}=202)$} & \multicolumn{2}{c}{ Sample 2 $(\mathrm{n}=246)$} \\
\hline IV / DV & BDI-II & SWLS & BDI-II & SWLS \\
\hline CD-RISC & $-.30^{* * *}$ & $.34^{* * *}$ & $-.37^{* * *}$ & $.56^{* * *}$ \\
BRS & $-.30^{* * *}$ & $.18^{*}$ & $-.29^{* * *}$ & $.13^{*}$ \\
& & & & \\
$R^{2}$ & .30 & .22 & .35 & .40 \\
$F$ & $41.77^{* * *}$ & $28.48^{* * *}$ & $66.50^{* * *}$ & $80.45^{* * *}$ \\
\hline
\end{tabular}

${ }^{*} p<.05 . \quad{ }^{* *} p<.01 . \quad{ }^{* * *} p<.001$.

Table 3. $R^{2}$ change in hierarchical regression analysis

\begin{tabular}{lcccc}
\hline & \multicolumn{2}{c}{ Sample 1 $(\mathrm{n}=202)$} & \multicolumn{2}{c}{ Sample 2 $(\mathrm{n}=246)$} \\
\hline IV / DV & BDI-II & SWLS & BDI-II & SWLS \\
\hline Step 1: CD-RISC & $.24^{* * *}$ & $.20^{* * *}$ & $.30^{* * *}$ & $.39^{* * *}$ \\
Step 2: BRS & $.05^{* * *}$ & $.02^{*}$ & $.05^{* * *}$ & $.01^{*}$ \\
& & & & $.21^{* * *}$ \\
Step 1: BRS & $.24^{* * *}$ & $.16^{* * *}$ & $.27^{* * *}$ & $.18^{* * *}$ \\
Step 2: CD-RISC & $.05^{* * *}$ & $.07^{* * *}$ & $.09^{* * *}$ & \\
\hline
\end{tabular}

${ }^{*} p<.05 . \quad{ }^{* *} p<.01 . \quad{ }^{* *} p<.001$.

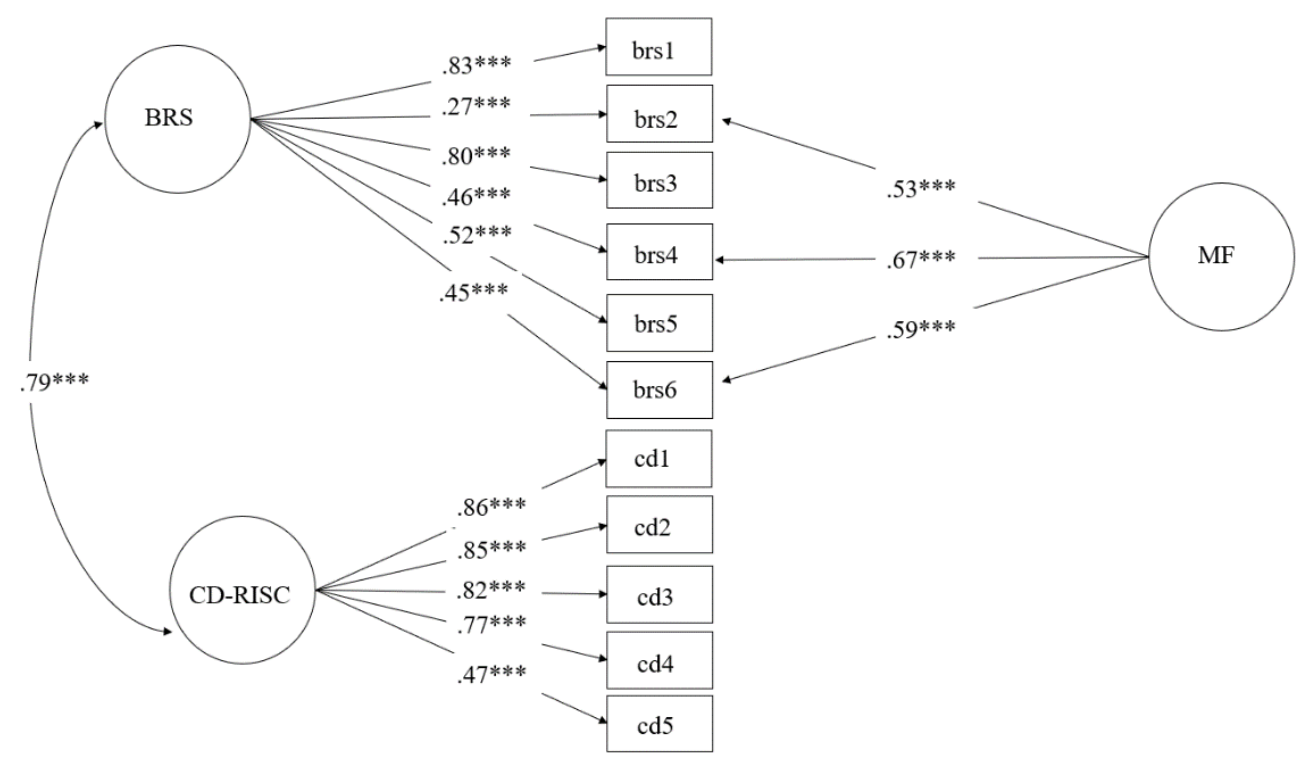

Figure 1. Standardized factor loadings from confirmatory factor analysis in Sample 1.

Note: $\mathrm{BRS}=$ brief resilience scale; $\mathrm{CD}$-RISC $=$ Connor-Davidson resilience scale; $\mathrm{MF}=$ method factor indicated by the three negatively worded items in BRS. Uniqueness variance of each indicators were skipped for simplicity.

${ }^{* * *} p<.001$. 


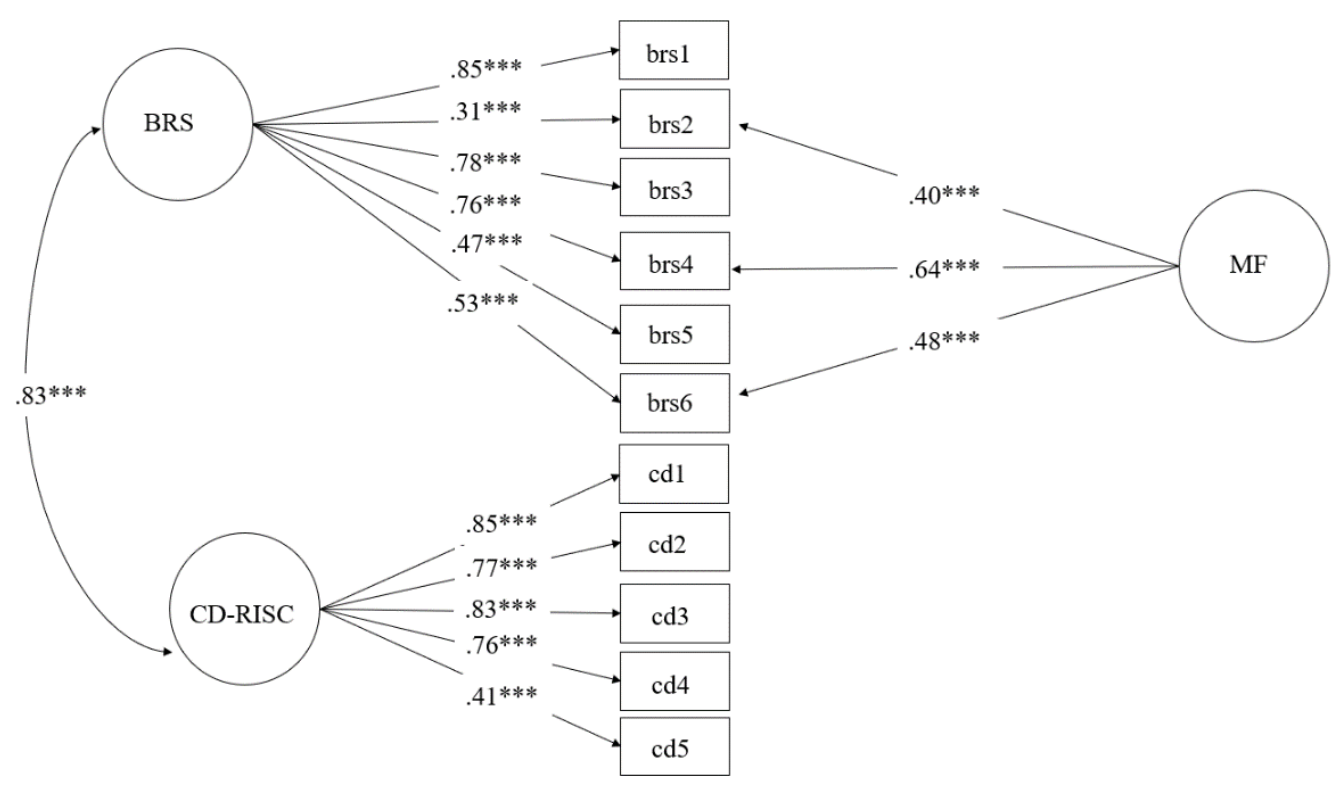

Figure 2. Standardized factor loadings from confirmatory factor analysis in Sample 2.

Note: $\mathrm{BRS}=$ brief resilience scale; $\mathrm{CD}$-RISC $=$ Connor-Davidson resilience scale; $\mathrm{MF}=$ method factor indicated by the three negatively worded items in BRS. Uniqueness variance of each indicators were skipped for simplicity.

${ }^{* * *} p<.001$.

\section{Discussion}

Across two samples, we found that the BRS and the CD-RISC, though highly related, have a unique predictive effect on measures of depression (i.e., BDI-II) and life satisfaction (i.e., SWLS). These findings suggest that BRS and CD-RISC capture different aspects of resilience and that both are important for wellbeing. While the findings support our hypotheses, two limitations should be noted. First, we only used samples of undergraduate students from Taiwan. Whether the findings can be generalized to samples from other countries should be further examined. Second, we only included measures of depression and life satisfaction as the two outcomes. Future studies should investigate whether the BRS and the CD-RISC also have unique predictive effects on other outcomes. Future studies are also encouraged to explore when the BRS or the CDRISC will be more important than the other to help individuals overcome and adapt to disruptions or stressful events. In other words, instead of investigating their differences with respect to resilient outcomes, identifying moderators or boundary conditions can also be the key to clarifying the differences between the BRS or the CD-RISC in capturing different aspects of resilience.

\section{Conclusions}

In this study, we provide evidence to suggest the need to clarify the differences between the BRS and the CD-RISC, the two widely used measurement for individual resilience. While both measurements are developed to measure individual resilience, the CD-RISC focuses on available resources in multiple aspects that help individuals recover from and adapt to disruptions and the BRS solely focuses on one's ability to bounce back. Researchers on individual resilience should pay attention to the differences between the two measurements and choose the appropriate one based on their research focus. 


\section{References}

1. Charney, D. S. (2004). Psychobiological mechanisms of resilience and vulnerability: implications for successful adaptation to extreme stress. American Journal of Psychiatry, 161(2), 195-216.

2. Masten, A. S. (2001). Ordinary magic: Resilience processes in development. American Psychologist, 56(3), $227-238$.

3. Pangallo, A., Zibarras, L., Lewis, R., \& Flaxman, P. (2015). Resilience through the lens of interactionism: A systematic review. Psychological Assessment, 27(1), 1-20.

4. Connor, K. M., \& Davidson, J. R. (2003). Development of a new resilience scale: The Connor-Davidson resilience scale (CDRISC). Depression and Anxiety, 18(2), 76-82.

5. Smith, B. W., Dalen, J., Wiggins, K., Tooley, E., Christopher, P., \& Bernard, J. (2008). The Brief Resilience Scale: Assessing the Ability to Bounce Back. International Journal of Behavioral Medicine, 15, 194-200.

6. Hilliard, M. E., McQuaid, E. L., Nabors, L., \& Hood, K. K. (2015). Resilience in youth and families living with pediatric health and developmental conditions: Introduction to the special issue on resilience. Journal of Pediatric Psychology, 40(9), 835-839.

7. Hobfoll, S. E. (2011). Conservation of resources theory: Its implication for stress, health, and resilience The Oxford handbook of stress, health, and coping. (pp. 127-147). New York, NY, US: Oxford University Press.

8. Rodríguez-Rey, R., Alonso-Tapia, J., \& Hernansaiz-Garrido, H. (2016). Reliability and validity of the Brief Resilience Scale (BRS) Spanish Version. Psychological Assessment, 28(5), e101-e110.

9. $\mathrm{Wu}, \mathrm{C} .-\mathrm{H}$. (2008). An examination of the wording effect in the Rosenberg self-esteem scale among culturally Chinese people. Journal of Social Psychology, 148, 535-552.

10. Tu, W.-M., Yan, M.-C., Wu, J.-R., Lin, M.-T., Wang, M.-H., Chan, F., \& Lee, B. (2017). Assessing resilience in persons with polio: A psychometric validation of the taiwanese version of the brief resilience scale [in Chinese]. Rehabilitation Counseling, 8, 1-18.

11. Wang, S.-H., Wang, T.-J., Hsu, S.-T., \& Wang, M.-H. (2017). Reliability and validity of the Chinese version of the ConnorDavidson resilience scale [in Chinese]. [Reliability and Validity of the Chinese Version of the Connor-Davidson Resilience Scale]. Rehabilitation Counseling, 8, 47-71.

12. Beck, A. T., Steer, R. A., \& Brown, G. K. (1996). Manual for the Beck DepressionInventory-II. San Antonio: Psychological Corporation.

13. Lu, M. L., Che, H. H., Chang, S. W., \& Shen, W. W. (2002). Reliability and validity of the Chinese version of the Beck Depression Inventory-II [In Chinese]. Taiwanese Journal of Psychiatry, 16, 301-310.

14. Diener, E., Emmons, R. A., Larsen, R. J., \& Griffin, S. (1985). The satisfaction with life scale. Journal of Personality Assessment, 49(1), 71-76.

15. Wu, C., \& Yao, G. (2006). Analysis of factorial invariance across gender in the Taiwan version of the satisfaction with life scale. Personality and Individual Differences, 40(6), 1259-1268.

16. Muthén, L. K., \& Muthén, B. O. (2012). Mplus User's Guide. Los Angeles, CA: Muthén \& Muthén. 